\title{
Rebound Tonometers versus Other Tonometers: Agreement among Readings and the Effects of Axial Length, Ocular Pulse Amplitude, and Central Corneal Thickness on Readings
}

\section{Seung Joo Ha}

Department of Ophthalmology, Soonchunhyang University Seoul Hospital, Soonchunhyang University College of Medicine, Seoul, Korea

\begin{abstract}
Objective: The aim of this study was to use intraocular pressure (IOP) measurements obtained via rebound tonometry (RBT, the Icare instrument), Goldmann applanation tonometry (GAT), non-contact tonometry (NCT), dynamic contour tonometry (DCT, PAS(AL), and the TonoPen to investigate the consistency of readings among methods and the influence of ocular pulse amplitude $(\mathrm{OPA})$, axial length (AL), and central corneal thickness (CCT) on RBT data.

Methods: We prospectively studied 123 eyes. IOP was measured via RBT, GAT, NCT, DCT, and the TonoPen. In addition, OPA was measured via DCT, AL, and CCT. Correlations among measurements using the various methods were evaluated, as were the effects of OPA, AL, and CCT on RBT data.

Results: RBT data were significantly correlated with data obtained via GAT, NCT, DCT, and the TonoPen; the highest correlation was with GAT. OPA was significantly correlated with IOP measured via GAT but not with IOP measured via RBT. Both AL and CCT were significantly correlated with IOP data obtained via RBT and GAT.

Conclusion: Significant correlations were evident among IOP measurements obtained via RBT and other tonometry methods. However, the influence of AL and CCT on IOP measurements obtained via RBT requires careful consideration and interpretation. Although the IOP values obtained by GAT were correlated with OPA values obtained by DCT, this was not true of IOP data obtained by RBT. This might be associated with characteristic of RBT which has the relatively short corneal contact time.
\end{abstract}

Keywords: Rebound tonometer; Goldmann applanation tonometer; Dynamic contour tonometer; Non-contact tonometer; TonoPen; Ocular pulse amplitude

\section{INTRODUCTION}

Accurate estimation of intraocular pressure (IOP) over time is important for assessing the risk of developing glaucoma. Goldmann applanation tonometry (GAT) is generally considered to be the "gold standard" for measuring IOP [1]. However, the patient must be cooperative and must be able to assume a sitting posture. Thus, use of GAT is difficult in children [2] and patients who are bedridden [3]. Several different methods have been developed to overcome the disadvantages of GAT; these include newer electronic applanation tonometers and rebound tonometry (RBT) [4].

RBT has been reported to yield data comparable to those obtained using GAT, and is increasingly employed to screen glauco- ma patients. The method is easy to use, does not require topical anesthesia or application of a fluorescent dye, is portable, and yields reproducible measurements [5-7]. Measurement of IOP using a rebound tonometer minimizes corneal injury, and avoids the risk of cross-infection because of the disposable probes. Ultimately, RBT may be valuable for self-measurement of IOP [3]. In RBT, a solenoid launches a magnetized probe, whose motion is monitored during corneal impact [8]. The probe is rather light (24.0 $\mathrm{mg}$ ), and is fired repeatedly onto the eye 6 times per measurement. Probe deceleration is measured as the probe bounces back from the cornea, and the data are transformed into an IOP value in $\mathrm{mmHg}$, which is displayed on an inbuilt screen [9].

Indeed, the accuracy of one such RBT device, I-care, has been
Correspondence to: Seung Joo Ha

Department of Ophthalmology, Soonchunhyang University Seoul Hospital, 59 Daesagwan-ro, Yongsan-gu, Seoul 04401, Korea

Tel: +82-2-709-9354, Fax: +82-2-709-9355, E-mail: sjha@schmc.ac.kr

Received: May 31, 2018 / Accepted after revision: Jun. 15, 2018
(C) 2018 Soonchunhyang Medical Research Institute

This is an Open Access article distributed under the terms of the Creative Commons Attribution Non-Commercial License (http://creativecommons.org/licenses/by-nc/4.0/) 
shown to be similar to those of both the TonoPen and a noncontact tonometer, and is comparable to that of GAT in both adults and children [10-13]. Some evidence indicates that IOP measurements obtained using RBT correlate excellently with GAT data, although a tendency to overestimate IOP, compared to values obtained via GAT, has been indicated; this was dependent on central corneal thickness (CCT) [3]. In contrast, another previous study showed that IOP data obtained via RBT were influenced by corneal properties including hysteresis and the resistance factor, but not by CCT [14]. The effects of axial length (AL) and ocular pulse amplitude (OPA), both of which affect ocular rigidity, on IOP measurement, warrant further study [15-17]. Therefore, in the present study, we evaluated the accuracy of RBT in clinical practice by comparing data obtained using I-care with those of GAT, noncontact tonometry (NCT), dynamic contour tonometry (DCT), and the TonoPen. We routinely use all of these instruments in the Eye and Ear Center of Soonchunhyang University Hospital (SCHUH). Also, we analyzed the effects of CCT, AL, and OPA on IOP data obtained via RBT and GAT.

\section{MATERIALS AND METHODS}

In this cross-sectional, observational study, we analyzed data from 123 eyes of 62 patients (91 eyes with glaucoma and 32 without glaucoma) who visited our department between August 2015 and October 2015 and who gave written informed consent to participate in the study. Institutional Review Board (IRB) of SCHUH designate an activity in this study as exemption due to minimal risk procedures to subject according to SCHUH IRB exemption guidelines. All research was conducted according to the guidelines of the Declaration of Helsinki. The exclusion criteria were any corneal pathology (e.g., a corneal epithelial defect, scarring, edema, or a graft), a history of intraocular surgery, and/or ocular inflammation.

Before measuring IOP, all patients were examined to exclude those with corneal pathologies that had developed in the time since any prior baseline examination. Work-up included measurement of best-corrected visual acuity, IOP, OPA using DCT, CCT using corneal pachymetry (SP-3000 instrument; Tomey, Nagoya, Japan), AL estimation (Hi-scan A/B scanning system; Optikon, Rome, Italy), and examination of the anterior and posterior segments of the eye via slit-lamp biomicroscopy.

All IOP measurements were performed on patients in the sitting position by three equally skilled ophthalmologists who had completed training in the use of all devices. The tests were always run in the following order to avoid any anomaly that might possibly arise if contact applanation tonometry reduced IOP: NCT (NT2000; NIDEK, Aichi, Japan), RBT (I-care Pro; TiolatOy, Helsinki, Finland), TonoPen (Tono-PenXL; Reichert, New York, NY, USA), GAT (AT900; Haag-Streit International, Koeniz, Switzerland), and DCT (PASCAL; Ziemer, Zurich, Switzerland). The RBT instrument had an adjustable forehead support, used to ensure accurate measurement of distance and correct alignment. Six measurements were obtained from each patient using this instrument, and the mean IOP was displayed on a liquid crystal display screen. Five to 10 minutes after RBT, GAT was performed after application of one drop of Paracaine (Proparacaine Hydrochloride $5 \mathrm{mg}$ / $\mathrm{mL}$; Hanmi Pharm, Seoul, Korea) and instillation of one drop of fluorescein (Haag Streit International) into the tear film.

Statistical analyses were performed using SPSS software ver. 14.0 (SPSS Inc., Chicago, IL, USA). Descriptive data were collected to establish the demographic characteristics of the study population. Then analyses of descriptive statistics, including the calculation of means and standard deviations of IOP measurements obtained using the various instruments, were conducted. The BlandAltman approach was used to assess agreement among instruments in terms of IOP measurement [18]. Linear regression analyses of Bland-Altman data were conducted to investigate whether IOP had been over- or underestimated within the measured range. Linear regression of the differences between RBT and GAT measurements, against estimated OPA values, was used to assess

Table 1. Descriptive statistics for all eyes; demographic characteristics of study subjects; measurements of IOP, axial length, and OPA; and pachymetry data

\begin{tabular}{lcccc}
\hline Variable & All patients & $\begin{array}{c}\text { Patients } \\
\text { with } \\
\text { glaucoma }\end{array}$ & $\begin{array}{c}\text { Patients } \\
\text { without } \\
\text { glaucoma }\end{array}$ & P-value \\
\hline No. of eyes/no. of patients & $123 / 62$ & $91 / 46$ & $32 / 16$ & \\
Age (yr) & $52.5 \pm 16.4$ & $56.3 \pm 13.7$ & $40.6 \pm 18.5$ & 0.001 \\
IOP (RBT) (mm Hg) & $13.9 \pm 2.8$ & $13.7 \pm 3.0$ & $14.5 \pm 2.1$ & 0.122 \\
IOP (GAT) (mm Hg) & $15.2 \pm 2.8$ & $15.3 \pm 2.8$ & $15.1 \pm 2.6$ & 0.750 \\
IOP (cGAT) (mm Hg) & $15.8 \pm 4.6$ & $15.9 \pm 7.9$ & $15.8 \pm 2.5$ & 0.887 \\
Axial length $(\mathrm{mm})$ & $24.4 \pm 1.7$ & $24.3 \pm 1.6$ & $24.7 \pm 2.0$ & 0.319 \\
CCT $(\mu \mathrm{m})$ & $526.7 \pm 39.5$ & $524.6 \pm 39.8$ & $533.0 \pm 38.8$ & 0.317 \\
OPA $(\mathrm{mm} \mathrm{Hg})$ & $2.6 \pm 0.9$ & $2.6 \pm 1.0$ & $2.5 \pm 0.8$ & 0.711 \\
\hline
\end{tabular}

Values are presented as mean \pm standard deviation, unless otherwise stated. IOP, intraocular pressure; OPA, ocular pulse amplitude; RBT, rebound tonometry; GAT, Goldmann applanation tonometry; cGAT, corrected GAT (calculated using the formula of Doughtly and Zaman); CCT, central corneal thickness. 
whether OPA was associated with any over- or underestimation of IOP measured via RBT.

\section{RESULTS}

Patient demographics are summarized in Table 1. We studied 35 female and 27 male patients (mean age, $52.5 \pm 16.4$ years; range, 20-79 years). A total of 91 eyes had glaucoma and 32 eyes did not. No significant difference was evident between those with and without glaucoma, except in terms of age. The mean IOP values obtained via RBT, GAT, NCT, DCT, and the TonoPen were 13.9 \pm $2.8 \mathrm{~mm} \mathrm{Hg}$ (range, 7-22.9 mm Hg), $15.2 \pm 2.8 \mathrm{~mm} \mathrm{Hg}$ (range, 9-23 mm Hg), $14.5 \pm 3.7$ mm Hg (range, 7-24 mm Hg), $17.7 \pm 2.8$ $\mathrm{mm} \mathrm{Hg}$ (range, 12-24 mm Hg), and 13.1 $\pm 2.4 \mathrm{~mm} \mathrm{Hg}$ (range, 9-21 mm Hg), respectively. The mean differences in IOP measurements between datas obtained via RBT and GAT, RBT and NCT,
RBT and DCT, and RBT and the TonoPen were $-1.1 \pm 3.6,-0.6 \pm$ $3.1,-3.8 \pm 2.9$, and $0.8 \pm 2.7 \mathrm{~mm} \mathrm{Hg}$, respectively.

Good agreement was evident between the IOP values yielded by RBT and GAT $(r=0.643, P<0.001)$, RBT and NCT $(r=0.633$, $\mathrm{P}<0.001)$, RBT and DCT $(\mathrm{r}=0.485, \mathrm{P}<0.001)$, and RBT and the TonoPen $(r=0.429, \mathrm{P}<0.001)$ (Fig. 1). RBT readings were within $\pm 3 \mathrm{~mm} \mathrm{Hg}$ of GAT values in $62.6 \%$ of all eyes, within $\pm 2 \mathrm{~mm}$ $\mathrm{Hg}$ in $48.8 \%$ of eyes, and within $\pm 1 \mathrm{~mm} \mathrm{Hg}$ in $26.8 \%$ eyes.

The mean CCT was $527.3 \pm 39.6 \mu \mathrm{m}$, ranging from 433 to $621 \mu$ $\mathrm{m}$. We found statistically significant agreement among measurements obtained using all tonometry methods (RBT: $\mathrm{P}=0.433$, $\mathrm{P}=0.000$; GAT: $\mathrm{P}=0.418, \mathrm{P}=0.000$; NCT: $\mathrm{P}=0.554, \mathrm{P}=0.000$; DCT: $\mathrm{P}=0.290, \mathrm{P}=0.001$; TonoPen: $\mathrm{P}=0.373, \mathrm{P}=0.000)$. IOP values obtained via RBT, GAT, DCT, and the TonoPen did not vary over a wide CCT range (RBT-GAT: $\beta=0.001, \mathrm{P}=0.828$; RBTDCT: $\beta=0.010, P=0.153$; RBT-Tonopen: $\beta=0.008, P=0.236$ ). The
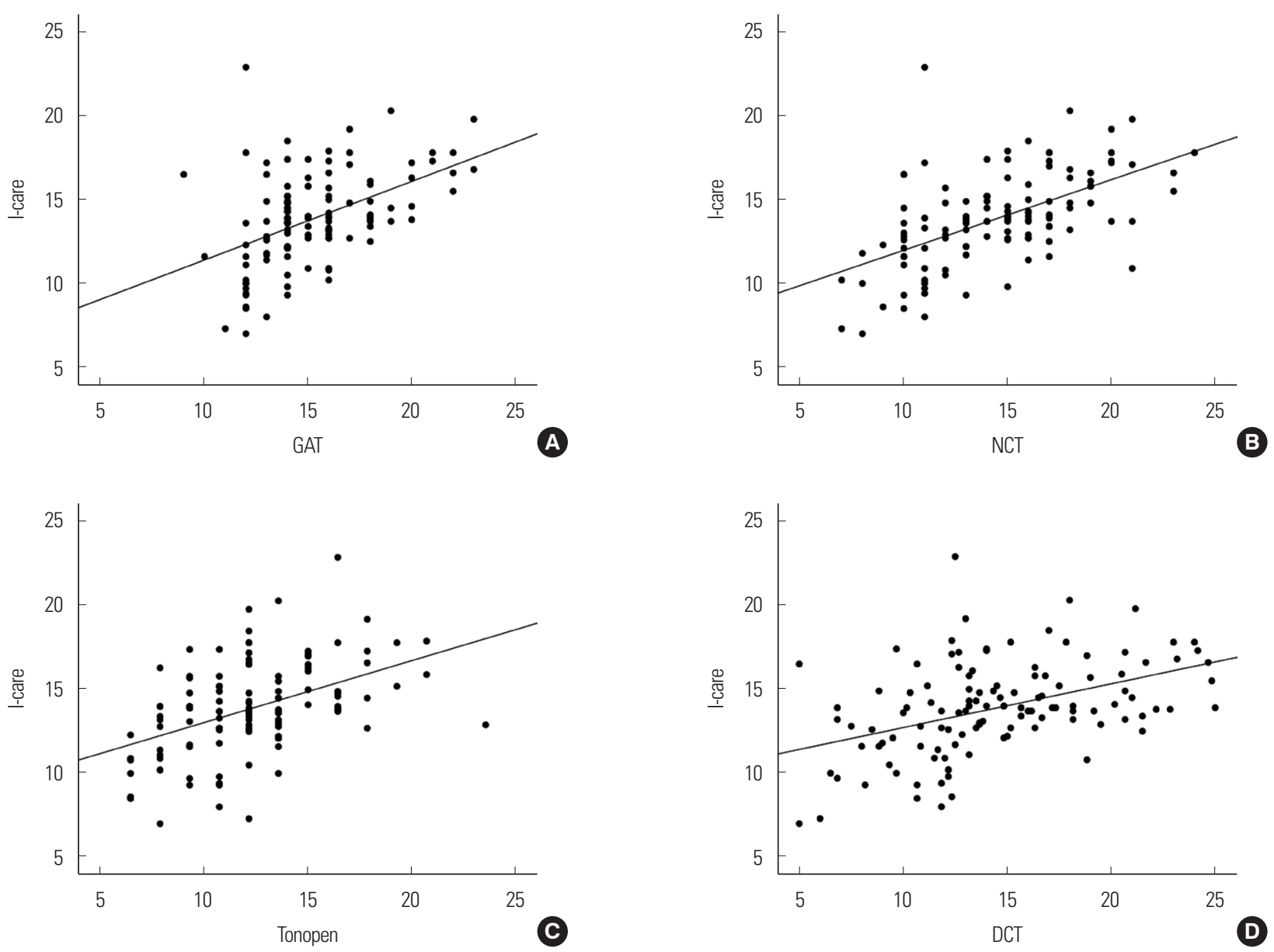

Fig. 1. (A-D) Correlations between intraocular pressure measurements and other measurements obtained using various tonometers, with reference to the 1 -care tonometer. The Pearson correlation coefficients (the r-values) were $0.643,0.633,0.485$, and 0.429 , respectively. All P-values $<0.001$. I-care, I-care rebound tonometer; GAT, Goldmann applanation tonometer; NCT, non-contact tonometer, DCT, dynamic contour tonometer. 
only observed difference was between RBT and NCT IOP values; this was statistically weak but nonetheless significant $\left(\mathrm{r}^{2}=0.073, \beta=\right.$ -0.023, $\mathrm{P}=0.003$ ) (Fig. 2).

The mean AL was $24.4 \pm 1.7 \mathrm{~mm}$, ranging from 21.9 to 29.5 $\mathrm{mm}$. Significant associations were found between AL and IOP measured via RBT $(P=0.257, P=0.005)$ and $D C T(P=0.293$, $\mathrm{P}=0.001)$, but not for the IOP values obtained via GAT $(\mathrm{P}=0.145$, $\mathrm{P}=0.121)$, NCT $(\mathrm{P}=0.151, \mathrm{P}=0.106)$, or the TonoPen $(\mathrm{P}=-0.002$, $\mathrm{P}=0.987$ ). IOP values did not differ significantly between RBT and GAT or between RBT and DCT over a wide range of AL values (RBT-GAT: $\beta=0.140, P=0.371$; RBT-DCT: $\beta=-0.080, P=$ 0.612) (Fig. 3).

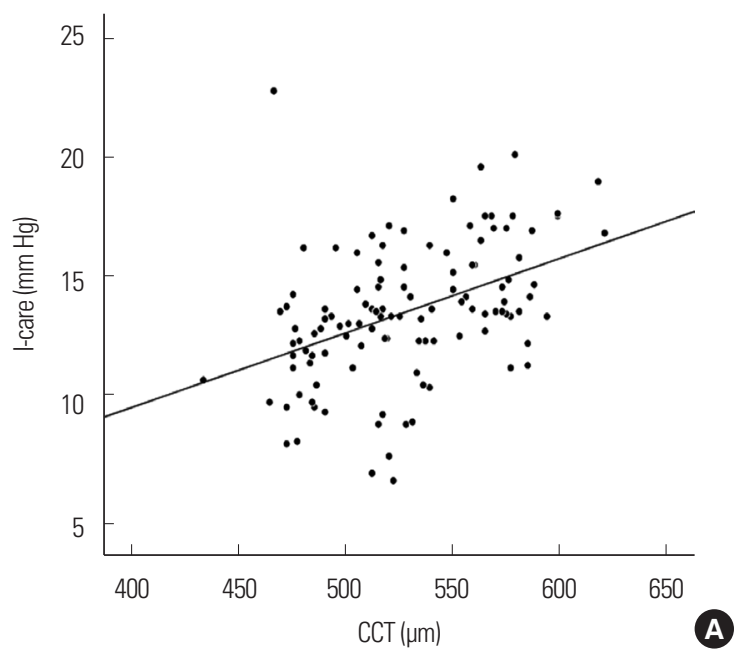

The mean OPA was $2.4 \pm 0.9 \mathrm{~mm} \mathrm{Hg}$, ranging from 1.2 to 6.5 $\mathrm{mm} \mathrm{Hg}$. The only significant correlation between OPA and IOP values was found with the IOP data from GAT $\left(\mathrm{r}^{2}=0.038, \mathrm{P}=\right.$ 0.032) (Fig. 4). The differences in IOP values between RBT and GAT were significantly correlated over the entire range of OPA values (RBT-GAT: $r^{2}=0.031, \beta=-0.523, P=0.05$ ) (Fig. 5).

The Bland-Altman scatter plot comparing data obtained via RBT and GAT showed that the two datasets were in reasonable agreement. The mean difference (bias) was $-1.1 \mathrm{~mm} \mathrm{Hg}$, the standard deviation was $2.6 \mathrm{~mm} \mathrm{Hg}$, and the $95 \%$ confidence interval was -6.3 to $4.1 \mathrm{~mm} \mathrm{Hg}$ (Fig. 6).

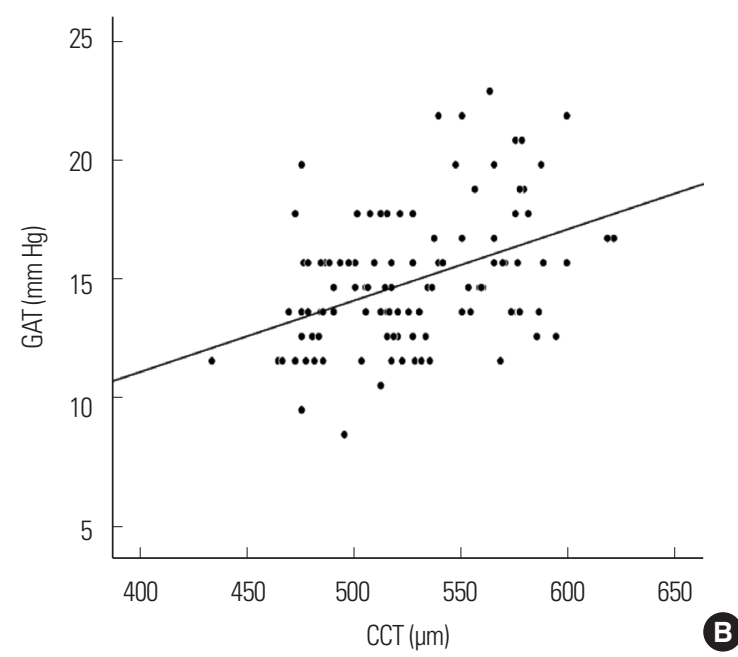

Fig. 2. (A, B) Representative figure of correlations between intraocular pressure and central corneal thickness measurements obtained using the rebound and Goldmann tonometers. CCT, central corneal thickness; I-care, I-care rebound tonometer; GAT, Goldmann tonometer.
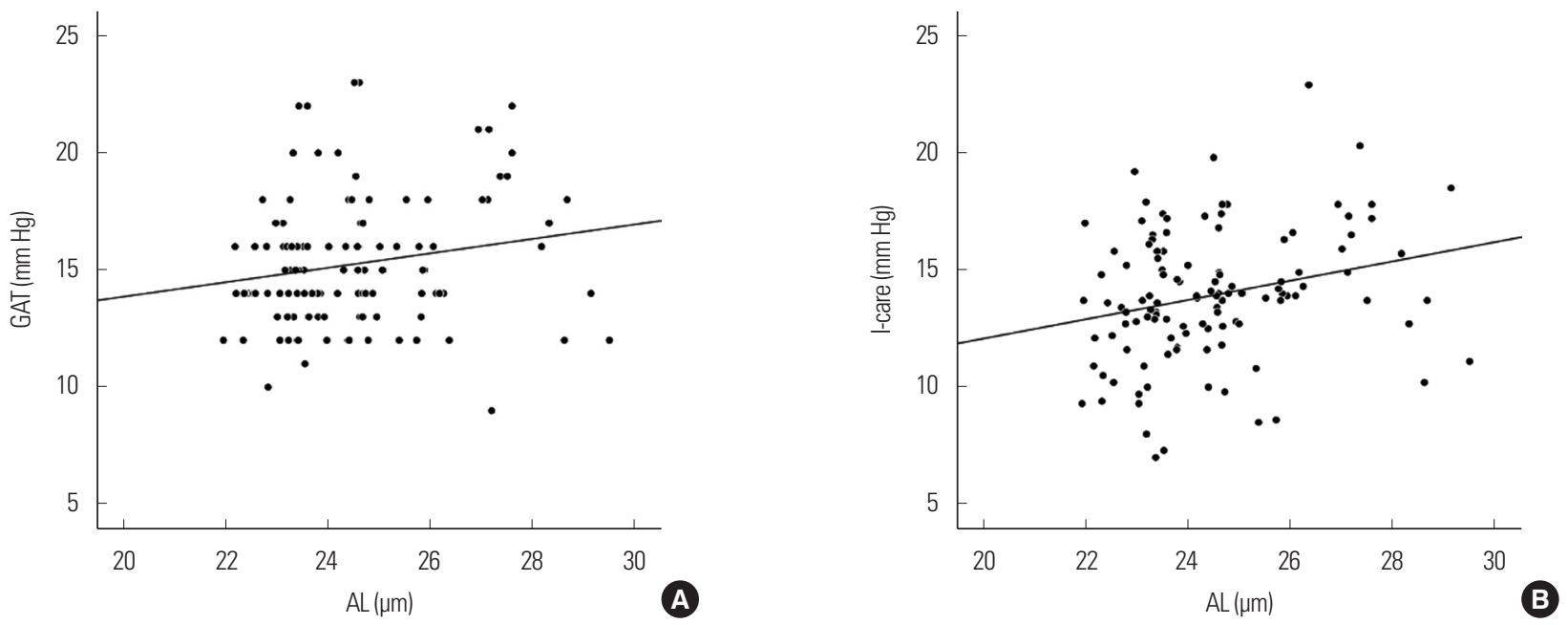

Fig. 3. (A, B) Correlations between intraocular pressure and axial length measurements obtained using the rebound and Goldmann tonometers. AL, axial length; I-care, I-care rebound tonometer; GAT, Goldmann tonometer. 

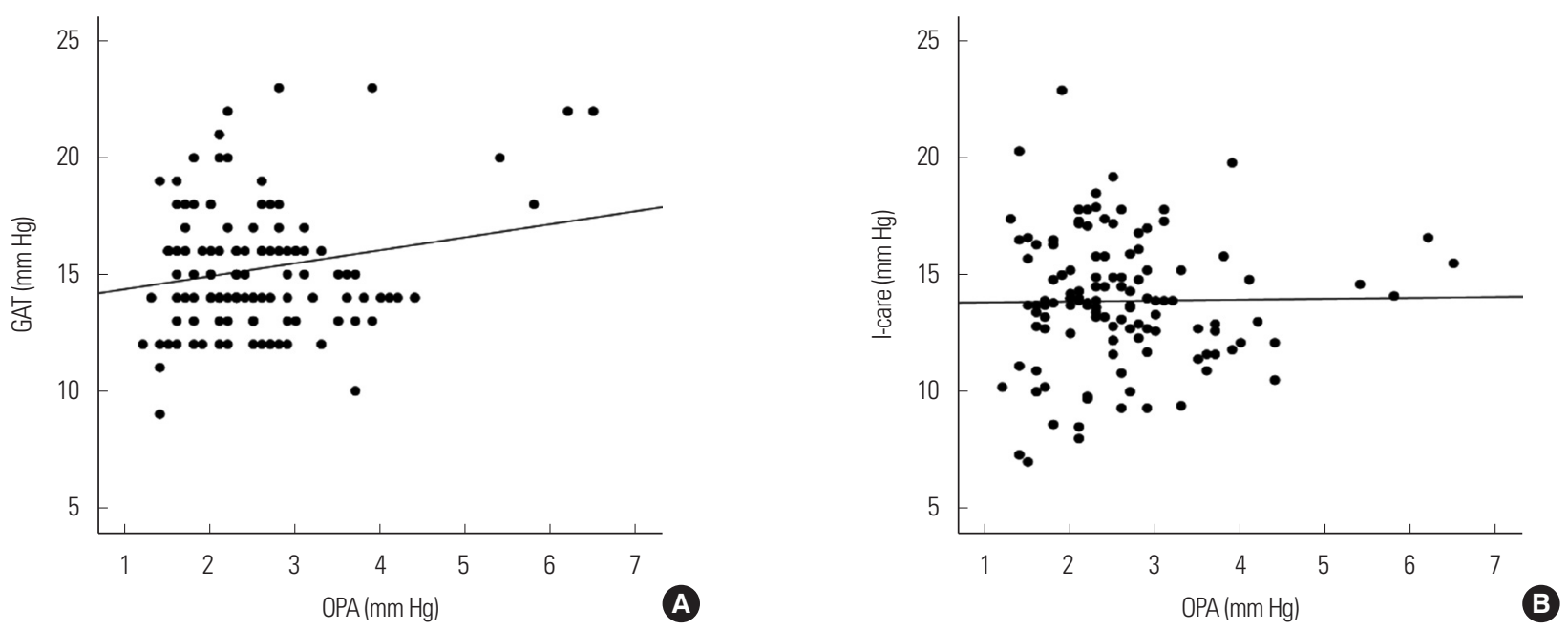

Fig. 4. (A, B) Correlations between measurements of intraocular pressure and OPA obtained using the rebound and Goldmann tonometers. OPA, ocular pulse amplitude; I-care, I-care rebound tonometer; GAT, Goldmann tonometer.

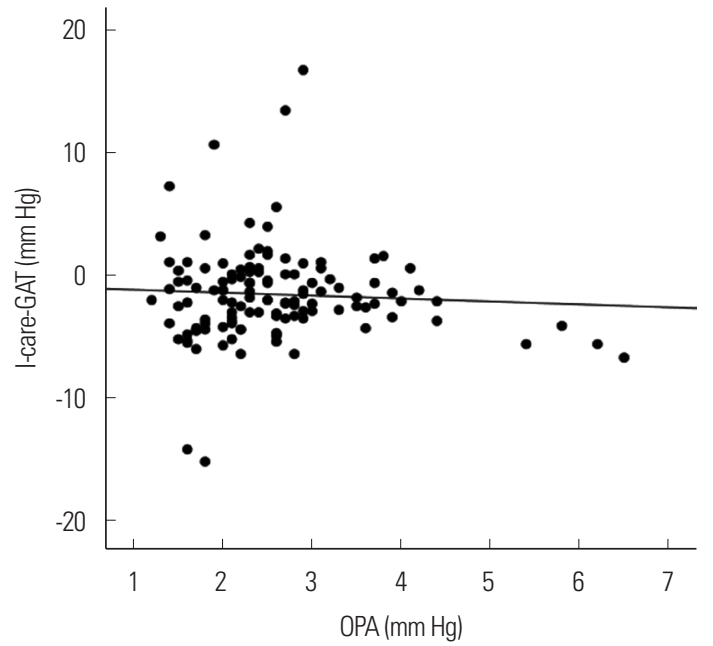

Fig. 5. Scatter plot of OPA versus the differences in IOP. $\triangle I O P$, each is an I-care value minus a GAT value; OPA, ocular pulse amplitude; I-care, I-care rebound tonometer; GAT, Goldmann tonometer.

\section{DISCUSSION}

RBT, also known as impact or dynamic tonometry, was first introduced by Obbink more than 75 years ago. It was modified in the 1960s by Dekking and Coster [19], and was further refined in 1997 by Kontiola [20]. The method employs a moving probe that collides with the eye; the parameters of the motion of the probe, which vary with eye pressure, are monitored and used to calculate IOP. The principal advantages of the method are that it is quick, easy to apply, and affordable. In 2000, Kontiola [21] introduced an improved and simpler RBT device that is better viewed as an in-

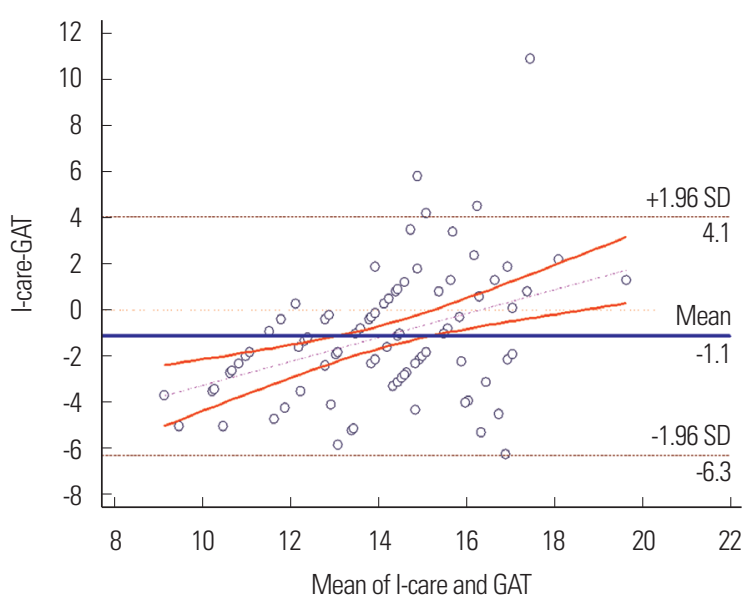

Fig. 6. Bland-Altman plot. The IOP differences between the RBT (I-care) and Goldmann tonometers are plotted against the means of the two measurements. IOP, intraocular pressure; OPA, ocular pulse amplitude; I-care, I-care rebound tonometer: GAT, Goldmann tonometer; SD, standard deviation.

duction-based impact tonometer. The prototype has yielded promising preliminary results $[5,21,22]$ and the instrument became commercially available as the I-care tonometer in 2003.

Several studies have compared the accuracies of RBT and GAT. In the clinic, RBT measures IOP accurately and reproducibly [2$4,7,9,12,23,24]$. However, although some studies that have included both healthy individuals and glaucoma patients have reported that RBT overestimates IOP compared to GAT [2,3,7,9,24], other studies have reported the opposite $[4,23,25]$. In the present study, the agreement between RBT and GAT IOP values was good, comparable to the results of previous studies, although the mean IOP 
measured via RBT $(13.9 \pm 2.8 \mathrm{~mm} \mathrm{Hg})$ was significantly lower than that measured via GAT $(15.2 \pm 2.8 \mathrm{~mm} \mathrm{Hg})(\mathrm{P}<0.001)$. Brusini et al. [4] found that mean IOP values obtained via GAT, corrected against CCT data, were nearly equal to IOP values obtained via RBT (the difference between the two mean values was $0.1 \mathrm{~mm} \mathrm{Hg}$ ); RBT was reported to have underestimated IOP by 1 $\mathrm{mm} \mathrm{Hg}$. Our mean corrected value obtained by GAT was $15.8 \pm$ $4.6 \mathrm{~mm} \mathrm{Hg}$; this is still higher than that yielded by RBT. Lee et al. [23] described a similar tendency evident when comparing the two methods (the mean RBT value minus the GAT value was -2.23 $\pm 3.16 \mathrm{~mm} \mathrm{Hg}$ and the mean RBT value minus the corrected GAT value was $-2.05 \pm 3.87 \mathrm{~mm} \mathrm{Hg}$ ).

Several explanations may be advanced as to why IOP measurements obtained via RBT may be higher than those yielded by other tonometries. A possible influence of topical anesthesia on IOP is one of the more popular explanations. Baudouin and Gastaud [26] reported a decrease in IOP both 1 and 5 minutes after instillation of one drop of the local anesthetics oxybuprocaine (mean IOP: $15.53 \mathrm{~mm} \mathrm{Hg}$ before application; $14.77 \mathrm{~mm} \mathrm{Hg} 1$ minute after application; $\mathrm{P}<0.001)$ and betoxycaine $(16.06 \mathrm{~mm} \mathrm{Hg}$ before, 15.70 $\mathrm{mm} \mathrm{Hg}$ at 1 minute; $\mathrm{P}=0.023)$. However, these decreases were not statistically significant. In our work, the time interval between instillation of anesthetic solution and measurement of IOP was less than 1 minute, so any effect of anesthesia on IOP would be expected to be negligible.

The Bland-Altman scatter plot revealed that, overall, the agreement between IOP measurements obtained via GAT and RBT was good. Linear regression analyses of the data revealed that the error was proportional over the range of IOPs measured (slope $=0.51$, $\mathrm{P}<0.001$ ) (Fig. 6). As shown in the graph, RBT values were lower than GAT values in the lower range of IOPs and higher than GAT values at higher IOPs (X-intercept, $16.2 \mathrm{~mm} \mathrm{Hg}$ ). Similarly, others have reported that RBT may overestimate IOP, compared to GAT values in patients with higher IOP values $(>21 \mathrm{~mm} \mathrm{Hg})[3,14,27,28]$.

Any effect of CCT on IOP measurements is based on the assumption that thinner corneas may be more deformable and therefore yield artificially low pressure measurements [3]. Reductions in recorded IOP (using GAT and RBT) have been reported after LASIK (laser-assisted in-situ keratomileusis) surgery [29,30] and (using GAT) after PRK (photorefractive keratectomy) [31]. In the present study, a weak but statistically significant correlation was evident between the data from all tonometries, including RBT, and CCT. However, the differences between RBT and GAT values did not vary over a wide range of CCT values. Some previous studies that have compared the influence of CCT on RBT and GAT data have indicated that RBT overestimates IOP relative to GAT as CCT increases [4,24,27], whereas other studies have found no such effect $[14,22]$. Recently developed RBT devices do not applanate the cornea, and thus theories seeking to explain why applanation tonometry yields IOP values that differ from data obtained via GAT cannot be applied to such instruments. Nevertheless, the rebound response of a probe may reflect the viscoelastic properties of the cornea, and thus might also be affected by the corneal thickness per se, at least to some extent.

We hypothesize that eyeball structure and property, including ocular rigidity, has a crucial effect on the deceleration of the probe as it bounces back off the cornea. Such rigidity, reflecting the relationship between pressure and volume changes in the eyeball, can be assessed by several methods including anterior chamber manometry; measuring changes in AL, OPA, and the fundus pulse; ultrasound elastography; and evaluating corneal hysteresis [16]. Both AL and OPA are relatively easy to measure and the data may be used to derive more accurate IOP measurements.

On purely physical grounds, one would expect a large globe with a larger surface area to accommodate deformation upon indentation or applanation more easily than a smaller globe. Thus, larger eyes should yield lower tonometric values than smaller ones at the same internal manometric pressure [32]. However, in the present study, the mean IOP values for GAT and RBT differed from those of other studies [32-34], and the data in both cases were positively correlated with AL. Leighton and Tomlinson [35] explored the relationship between ocular tension and eyeball AL in open-angle glaucoma patients, and found that those with small (large) eyeballs tended to have high (low) maximal applanation ocular tension. When myopia develops, the production of aqueous humor overwhelms the slightly subnormal outflow channels and thus the eyeball distends. As a result, both the corneal circumference and the antero-posterior extent of the corneal meshwork probably increase to enhance the cross-sectional area of the outflow channels. Thinning of the ciliary body and the sclera may also increase uveoscleral bulk flow. Neither of these changes fully compensates for the higher level of aqueous humor, and thus IOP rises in myopic eyes [36]. Hence, patients with relatively large eyeballs, who produce large amounts of aqueous humor as myopia develops, may yield high applanation and rebound IOP values. This was evident in the glaucoma patients in the present study: the 
positive correlation between AL and RBT was 0.323 , but was only 0.047 for normal eyes. A large eyeball in a restricted orbit space would be under more pressure than would a small eyeball; this may also explain the positive correlation noted between AL and IOP. Furthermore, among small eyeballs, only those with an extremely short AL (e.g., eyes with nanophthalmos), thick sclera, and/or an anteriorly rotated ciliary body would show such a tendency.

However, our first hypothesis, that a longer AL (such as may be noted in myopic individuals) seems to serve as an indicator of a less-rigid eye or cornea, remains persuasive. Ishii et al. [36] evaluated the effects of eyeball structure on OPA, measured via DCT, and reported that OPA was significantly positively correlated with IOP data from GAT, DCT, and RBT. However, the relationship between the DCT data and OPA was only weakly significant. In the present study, the correlation between OPA and GAT data was strongest, that between OPA and DCT data was weakest, and there was no significant relationship between OPA and the RBT data. Kaufmann et al. [37] found that a lower OPA was associated with a longer AL, as did we in the present study (the OPA-AL correlation efficient was $r=-0.343, P=0.000$ ). These findings suggest that OPA serves as an indicator of ocular rigidity and may explain the differences in IOP values yielded by the various instruments. The difference in IOP data yielded by RBT and GAT was significantly associated with OPA, suggesting that IOP is possibly underestimated when GAT is used to evaluate highly myopic eyes. Our results are compatible with a previous study [38]. However, the possibility remains that our findings may in fact be explained by the short contact time between the probe and the cornea during RBT; this time is shorter than that of any other tonometry method. Indeed, multivariate analysis revealed that the factor that most significantly influenced the RBT data was CCT $(r=0.522, \mathrm{P}=$ 0.000); other factors including AL and OPA were not of importance in this regard $(\mathrm{r}=0.172, \mathrm{P}=0.197 ; \mathrm{r}=-0.010, \mathrm{P}=0.942$, respectively).

In conclusion, significant correlations were evident among IOP measurements obtained via RBT and other systems. However, both AL and CCT require careful consideration when IOP measurements are interpreted. Despite the observed correlation between OPA and GAT-derived IOP data, we found no correlation between OPA and RBT-derived IOP data. This may indicate that the two IOP measurement methods are differentially influenced by the extent of ocular rigidity, but it remains possible that the ex- planation may in fact lie in the relatively short contact time between the probe and the cornea during RBT.

\section{ACKNOWLEDGMENTS}

This work was supported by the Soonchunhyang University Research Fund.

\section{REFERENCES}

1. Kass MA. Standardizing the measurement of intraocular pressure for clinical research: guidelines from the Eye Care Technology Forum. Ophthalmology 1996;103:183-5.

2. Suman S, Agrawal A, Pal VK, Pratap VB. Rebound tonometer: ideal tonometer for measurement of accurate intraocular pressure. J Glaucoma 2014;23:633-7.

3. Rosentreter A, Jablonski KS, Mellein AC, Gaki S, Hueber A, Dietlein TS. A new rebound tonometer for home monitoring of intraocular pressure. Graefes Arch Clin Exp Ophthalmol 2011;249:1713-9.

4. Brusini P, Salvetat ML, Zeppieri M, Tosoni C, Parisi L. Comparison of ICare tonometer with Goldmann applanation tonometer in glaucoma patients. J Glaucoma 2006;15:213-7.

5. Kontiola A, Puska P. Measuring intraocular pressure with the Pulsair 3000 and Rebound tonometers in elderly patients without an anesthetic. Graefes Arch Clin Exp Ophthalmol 2004;242:3-7.

6. Rehnman JB, Martin L. Comparison of rebound and applanation tonometry in the management of patients treated for glaucoma or ocular hypertension. Ophthalmic Physiol Opt 2008;28:382-6.

7. Kim KN, Jeoung JW, Park KH, Yang MK, Kim DM. Comparison of the new rebound tonometer with Goldmann applanation tonometer in a clinical setting. Acta Ophthalmol 2013;91:e392-6.

8. Nakamura M, Darhad U, Tatsumi Y, Fujioka M, Kusuhara A, Maeda H, et al. Agreement of rebound tonometer in measuring intraocular pressure with three types of applanation tonometers. Am J Ophthalmol 2006; 142:332-4.

9. Munkwitz S, Elkarmouty A, Hoffmann EM, Pfeiffer N, Thieme H. Comparison of the iCare rebound tonometer and the Goldmann applanation tonometer over a wide IOP range. Graefes Arch Clin Exp Ophthalmol 2008;246:875-9.

10. Cook JA, Botello AP, Elders A, Fathi Ali A, Azuara-Blanco A, Fraser C, et al. Systematic review of the agreement of tonometers with Goldmann applanation tonometry. Ophthalmology 2012;119:1552-7.

11. Iliev ME, Goldblum D, Katsoulis K, Amstutz C, Frueh B. Comparison of rebound tonometry with Goldmann applanation tonometry and correlation with central corneal thickness. Br J Ophthalmol 2006;90:833-5.

12. Martinez-de-la-Casa JM, Garcia-Feijoo J, Castillo A, Garcia-Sanchez J. Reproducibility and clinical evaluation of rebound tonometry. Invest Ophthalmol Vis Sci 2005;46:4578-80.

13. Sahin A, Basmak H, Yildirim N. The influence of central corneal thickness and corneal curvature on intraocular pressure measured by tonopen and rebound tonometer in children. J Glaucoma 2008;17:57-61.

14. Chui WS, Lam A, Chen D, Chiu R. The influence of corneal properties on rebound tonometry. Ophthalmology 2008;115:80-4.

15. Ishii K, Mori M, Oshika T. An evaluation of the effects of eyeball structure on ocular pulse amplitude in healthy subjects. Int Ophthalmol 2012; 
32:553-7.

16. Detorakis ET, Pallikaris IG. Ocular rigidity: biomechanical role, in vivo measurements and clinical significance. Clin Exp Ophthalmol 2013;41: 73-81.

17. Dastiridou AI, Ginis H, Tsilimbaris M, Karyotakis N, Detorakis E, Siganos C, et al. Ocular rigidity, ocular pulse amplitude, and pulsatile ocular blood flow: the effect of axial length. Invest Ophthalmol Vis Sci 2013;54: 2087-92.

18. Bland JM, Altman DG. Statistical methods for assessing agreement between two methods of clinical measurement. Lancet 1986;1:307-10.

19. Dekking HM, Coster HD. Dynamic tonometry. Ophthalmologica 1967; 154:59-74.

20. Kontiola A. A new electromechanical method for measuring intraocular pressure. Doc Ophthalmol 1996-1997;93:265-76.

21. Kontiola AI. A new induction-based impact method for measuring in traocular pressure. Acta Ophthalmol Scand 2000;78:142-5.

22. Kontiola AI, Goldblum D, Mittag T, Danias J. The induction/impact tonometer: a new instrument to measure intraocular pressure in the rat. Exp Eye Res 2001;73:781-5.

23. Lee K, Lee JY, Moon JI, Park MH. Comparison of Icare rebound tonometer with Goldmann applanation tonometry. J Korean Ophthalmol Soc 2013;54:296-302.

24. Lee J, Seong M, Kang M, Cho H, Lee Y. Comparison of rebound tonometer, non-contact tonometer, Goldmann applanation tonometer and the relationship to central corneal thickness. J Korean Ophthalmol Soc 2012; 53:988-95.

25. Salvetat ML, Zeppieri M, Miani F, Tosoni C, Parisi L, Brusini P. Comparison of iCare tonometer and Goldmann applanation tonometry in normal corneas and in eyes with automated lamellar and penetrating keratoplasty. Eye (Lond) 2011;25:642-50.

26. Baudouin C, Gastaud P. Influence of topical anesthesia on tonometric values of intraocular pressure. Ophthalmologica 1994;208:309-13.

27. Pakrou N, Gray T, Mills R, Landers J, Craig J. Clinical comparison of the Icare tonometer and Goldmann applanation tonometry. J Glaucoma 2008;17:43-7.
28. Garcia-Resua C, Gonzalez-Meijome JM, Gilino J, Yebra-Pimentel E. Accuracy of the new ICare rebound tonometer vs. other portable tonometers in healthy eyes. Optom Vis Sci 2006;83:102-7.

29. Lam AK, Wu R, Wang Z, Woo V, Chan E, Tam K, et al. Effect of laser in situ keratomileusis on rebound tonometry and Goldmann applanation tonometry. J Cataract Refract Surg 2010;36:631-6.

30. Emara B, Probst LE, Tingey DP, Kennedy DW, Willms LJ, Machat J. Correlation of intraocular pressure and central corneal thickness in normal myopic eyes and after laser in situ keratomileusis. J Cataract Refract Surg 1998;24:1320-5.

31. Schipper I, Senn P, Oyo-Szerenyi K, Peter R. Central and peripheral pressure measurements with the Goldmann tonometer and Tono-Pen after photorefractive keratectomy for myopia. J Cataract Refract Surg 2000;26: 929-33.

32. Mark HH, Robbins KP, Mark TL. Axial length in applanation tonometry. J Cataract Refract Surg 2002;28:504-6.

33. Armaly MF. On the distribution of applanation pressure: I. statistical features and the effect of age, sex, and family history of Glaucoma. Arch Ophthalmol 1965;73:11-8.

34. Goedbloed J, Schappert-Kimmijser J, Donders PC, Henkes HE, van der Heuvel J, Hoeksema BL, et al. Frequency distribution of the intraocular pressure in the Netherlands. Ophthalmologica 1961;141:481-8.

35. Leighton DA, Tomlinson A. Ocular tension and axial length of the eyeball in open-angle glaucoma and low tension glaucoma. $\mathrm{Br} \mathrm{J} \mathrm{Ophthalmol}$ 1973; 57:499-502.

36. Ishii K, Mori M, Oshika T. An evaluation of the effects of eyeball structure on ocular pulse amplitude in healthy subjects. Int Ophthalmol 2012; 32:553-7.

37. Kaufmann C, Bachmann LM, Robert YC, Thiel MA. Ocular pulse amplitude in healthy subjects as measured by dynamic contour tonometry. Arch Ophthalmol 2006;124:1104-8

38. Wang J, Cayer MM, Descovich D, Kamdeu-Fansi A, Harasymowycz PJ, $\mathrm{Li} \mathrm{G}$, et al. Assessment of factors affecting the difference in intraocular pressure measurements between dynamic contour tonometry and goldmann applanation tonometry. J Glaucoma 2011;20:482-7. 